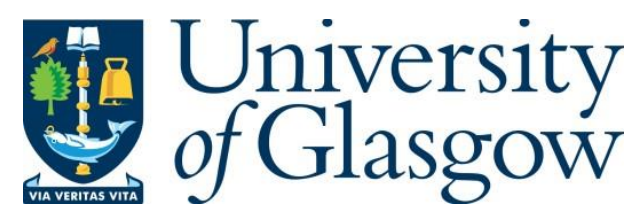

Ridler, N. M., Clarke, R. G., Li, C. and Salter, M. J. (2019) Strategies for traceable submillimeter-wave vector network analyzer measurements. IEEE Transactions on Terahertz Science and Technology, 9(4), pp. 392-398.

There may be differences between this version and the published version. You are advised to consult the publisher's version if you wish to cite from it.

http://eprints.gla.ac.uk/183896/

Deposited on: 12 April 2019

Enlighten - Research publications by members of the University of Glasgow http://eprints.gla.ac.uk 


\title{
Strategies for Traceable Submillimeter-wave Vector Network Analyzer Measurements
}

\author{
Nick M. Ridler, Fellow, IEEE, Roland G. Clarke, Member, IEEE, Chong Li, Senior Member, IEEE, \\ and Martin J. Salter, Member, IEEE
}

\begin{abstract}
This paper presents a strategy for achieving metrological traceability using vector network analyzers (VNAs) at submillimeter-wave frequencies $(300$ to $3000 \mathrm{GHz})$. The strategy includes the use of traceable calibration techniques designed for operation at these frequencies. Slight, but significant, physical differences between the waveguide line standards, used during calibration, are accommodated by applying a weighting technique to combine results using different calibration lines. Measurement uncertainty is assessed by analyzing replicate measurement data, to take account of the different waveguide interface interactions that occur when the line standards are connected to the VNA. The strategy is illustrated using measurements made in the WM-250 (750 GHz to $1100 \mathrm{GHz})$ waveguide band.
\end{abstract}

Index Terms-Measurement traceability, vector network analyzer, calibration, waveguide, submillimeter-wave measurements, measurement uncertainty

\section{INTRODUCTION}

A recent technology roadmap [1] highlighted the rapid growth in exploitation of technology that uses the submillimeter-wave (i.e. terahertz) part of the electromagnetic spectrum. This included the development of electronic components (including semiconductors) that operate at these frequencies. Several recent large research activities have concentrated on the development of these semiconductor device technologies. For example, the USA DARPA THz Electronics Program [2] developed electronic components (i.e. transistors, etc) to enable electronic circuits to be realized for communications applications - specifically, at three frequencies $(670 \mathrm{GHz}, 850 \mathrm{GHz}$ and $1030 \mathrm{GHz})$ corresponding to "propagation windows" (where atmospheric attenuation is relatively low) in this part of the electromagnetic spectrum. A related research activity in Europe was the 'dotseven' EU project [3], which developed electronic component capabilities to $700 \mathrm{GHz}$.

The development of such devices has driven the need for accurate and reliable measurement capabilities to enable these devices to be tested and characterized for use in practical circuits, such as amplifiers, transceivers, etc. This paper

This work was part-supported by the 2017-2020 National Measurement System (NMS) Program of the U.K. government's Department for Business, Energy \& Industrial Strategy (BEIS).

N. M. Ridler and M. J. Salter are with the National Physical Laboratory, Teddington, U.K. (e-mail: nick.ridler@npl.co.uk, martin.salter@npl.co.uk). describes some recent work on establishing a strategy for providing traceable measurement capabilities at these frequencies - specifically, in waveguide bands covering the whole submillimeter-wave frequency region (i.e. from $300 \mathrm{GHz}$ to $3000 \mathrm{GHz}$ ). This frequency range includes all the above frequencies of interest. Implementation of the strategy is shown with some example measurement results obtained in the WM-250 waveguide band (from $750 \mathrm{GHz}$ to $1100 \mathrm{GHz}$ ).

\section{CAlibration STANDARDS AND TEChNiQues}

At radio, microwave and millimeter-wave frequencies, measurements of electronic devices are usually made using vector network analyzers (VNAs). The accuracy of the VNA measurements is assured through the use of reliable calibration techniques and reference standards that are traceable to the International System of units (SI) [4]. The most appropriate standards for this purpose are usually sections of precision transmission lines. For example, air dielectric coaxial lines have been used as such standards at frequencies up to $65 \mathrm{GHz}$ [5] and sections of air-filled rectangular metallic waveguide have been used at frequencies up to $110 \mathrm{GHz}$ [6]. In both cases, these standards are used in conjunction with the Thru-Reflect-Line (TRL) [7] and Line-Reflect-Line (LRL) [8] calibration techniques to establish measurement traceability for the calibrated VNA. Measurement traceability is achieved by performing dimensional measurements on these Line standards and linking these dimensional measurements to the SI base unit, the meter.

These TRL and LRL calibration techniques can be extended to higher frequencies (i.e. above $110 \mathrm{GHz}$ ), again, using precision sections of waveguide as the reference standards. However, consideration is needed regarding the shorter wavelengths that occur at these higher frequencies.

Firstly, the TRL and LRL calibration schemes require very accurate dimensional characterization of the first of these standards because the electrical characteristics (i.e. S-parameters) of the first standard needs to be assumed to be fully known [8]. In the case of TRL, the first standard is a Thru connection which does not involve the use of a physical length of line - it is achieved by simply joining the two VNA test ports

R. G. Clarke is with the University of Leeds, Leeds, U.K. (e-mail: r.g.clarke@leeds.ac.uk).

C. $\mathrm{Li}$ is with the University of Glasgow, Glasgow, U.K. (e-mail: chong.li@glasgow.ac.uk). 
together. However, in the case of LRL, the electrical characteristics of the first Line standard need to be assumed to be fully known. Such a characterization is usually performed using dimensional measurements. Measured imperfections in the dimensions of the waveguide Line standard impact the quality of the LRL calibration. At low frequencies, where wavelengths are relatively long, the impact of these dimensional imperfections will be relatively small. However, at high frequencies (i.e. above $110 \mathrm{GHz}$ ) these dimensional imperfections in the Line standard can no longer be ignored. For this reason, the LRL calibration technique is not recommended for high precision measurement applications above $110 \mathrm{GHz}$.

Measurement traceability has previously been implemented at these high millimeter-wave frequencies using a modified version of the TRL calibration technique [9-11]. In a conventional TRL calibration, the Line standard consists of a section of line that provides a change in the transmission phase, with respect to the Thru connection, of approximately $90^{\circ}-$ i.e. $1 / 4$-wavelength ( $1 / 4$-wave) - at frequencies around the middle of the waveguide band. However, at higher frequencies (where wavelengths are small), this requires the use of a line with very short length (for example, a $1 / 4$-wave line in WM-250 waveguide, which operates from $750 \mathrm{GHz}$ to $1,100 \mathrm{GHz}$, is only $108 \mu \mathrm{m}$ in length). Such a short section of line can easily become damaged during use and so such lines are not considered suitable for this role. The modified TRL technique in [9-11], for calibrations over the frequency range $110 \mathrm{GHz}$ to $330 \mathrm{GHz}$, used two sections of waveguide (for each waveguide band) providing a phase change of $270^{\circ}$ (i.e. $3 / 4$-wave) at two different frequencies across the waveguide band - one in the lower half of the band and one in the upper half of the band. This technique has been described in detail in [12], which presents generalized calibration strategies for VNAs at these millimeter-wave frequencies. Reference [12] also described extending these strategies to submillimeter-wave frequencies by using three sections of waveguide providing a phase change of $450^{\circ}$ (i.e. 5/4-wave) at three different frequencies across a given waveguide band. However, it is quite time-consuming to connect the three lines that are needed with this technique to cover the full bandwidth of each waveguide band (and also increases measurement uncertainty due to misalignment) and so this technique has not been implemented routinely at these submillimeter-wave frequencies.

An alternative strategy is to keep to using just two $3 / 4$-wave lines at these submillimeter-wave frequencies. According to [12], the following steps are used to determine the lengths of the two TRL lines to achieve 3/4-wave TRL calibration in any given waveguide band:

Step 1: determine the length of the first line, $l_{1}$, as follows:

$$
l_{1}=\frac{\lambda_{\mathrm{g}(\max )}}{360} \times \varphi_{\min }
$$

where $\lambda_{\mathrm{g}(\max )}$ is the guide wavelength at the lowest recommended frequency in the waveguide band, and $\varphi_{\min }$ is the minimum recommended phase change.
The TRL calibration scheme works optimally when the difference in phase between the Thru and the Line standards is $(2 n+1) \lambda / 4$ and fails completely when this phase difference is $(2 n) \lambda / 4$ (where, in both cases, $n=0,1,2, \ldots$ ). Therefore, the lengths of TRL lines are chosen to avoid providing phase differences that are close to these calibration failure points. In [12], a minimum phase difference criterion was set such that all phase differences were at least $30^{\circ}$ away from the calibration failure points. For a $1 / 4$-wave TRL calibration, these calibration failure points occur at $0^{\circ}$ and $180^{\circ}$. For a $3 / 4$-wave TRL calibration, these calibration failure points occur at $180^{\circ}$ and $360^{\circ}$. Therefore, when implementing a $3 / 4$-wave TRL calibration procedure, phase changes are designed to be at least $30^{\circ}$ greater than $180^{\circ}$ (i.e. $>210^{\circ}$ ) and at least $30^{\circ}$ less than $360^{\circ}$ (i.e. $<330^{\circ}$ ). Therefore, $\varphi_{\min }=210^{\circ}$ in equation (1):

$$
l_{1}=\frac{\lambda_{\mathrm{g}(\max )}}{360} \times \varphi_{\min }=\frac{\lambda_{\mathrm{g}(\max )}}{360} \times 210 \approx 0.583 \lambda_{\mathrm{g}(\max )}
$$

Step 2: Establish the useable upper frequency limit for $l_{1}$, i.e. the frequency at which the maximum phase change, $\varphi_{\max }$ $\left(=330^{\circ}\right)$, occurs. This is achieved by determining the guide wavelength, $\lambda_{\mathrm{g}(f)}$, at the frequency, $f$, at which the maximum phase change occurs:

$$
\lambda_{\mathrm{g}(f)}=\frac{360 \times l_{1}}{\varphi_{\max }}=\frac{360}{330} \times l_{1} \approx 1.091 l_{1}
$$

and then determining $f$ using:

$$
f=\frac{\sqrt[v]{1+\left(\frac{\lambda_{\mathrm{g}(f)}}{\lambda_{0}}\right)^{2}}}{\lambda_{\mathrm{g}(f)}}
$$

where $v$ is the speed of electromagnetic waves in the air-filled waveguide and $\lambda_{0}$ is the waveguide cut-off wavelength.

Step 3: determine the length of the second line, $l_{2}$, which gives the maximum phase change, $\varphi_{\max }\left(=330^{\circ}\right)$, at the maximum recommended frequency for the given waveguide band;

$$
l_{2}=\frac{\lambda_{\mathrm{g}(\min )}}{360} \times \varphi_{\max }=\frac{\lambda_{\mathrm{g}(\min )}}{360} \times 330 \approx 0.917 \lambda_{\mathrm{g}(\min )}
$$

where $\lambda_{\mathrm{g}(\min )}$ is the minimum guide wavelength, which occurs at the highest recommended frequency in the waveguide band.

Step 4: establish the useable lower frequency limit for $l_{2}$, i.e. the frequency at which $\varphi_{\min }\left(=210^{\circ}\right)$ occurs. This is achieved by determining the guide wavelength, $\lambda_{\mathrm{g}(f)}$, at the frequency, $f$, at which the maximum phase change occurs:

$$
\lambda_{\mathrm{g}(f)}=\frac{360 \times l_{2}}{\varphi_{\min }}=\frac{360}{210} \times l_{2} \approx 1.714 l_{2}
$$

then calculating $f$ using equation (4).

To illustrate this procedure, Table 1 shows $3 / 4$-wave TRL line choices for standardized waveguide bands [13] in the submillimeter-wave region - i.e. from $330 \mathrm{GHz}$ to $3300 \mathrm{GHz}$. 
(Note: at the time of writing this paper, VNAs are not commercially available in waveguide bands beyond WM-164.)

TABLE I

3/4-WAVE TRL CALIBRATION USING TWO LINES

\begin{tabular}{|c|c|c|c|c|c|}
\hline $\begin{array}{c}\text { Waveguide } \\
\text { band }\end{array}$ & $\begin{array}{c}\text { Waveguide } \\
\text { frequency } \\
\text { range } \\
(\mathrm{GHz})\end{array}$ & $\begin{array}{c}l_{1} \\
(\mu \mathrm{m})\end{array}$ & $\begin{array}{c}l_{1} \\
\text { frequency } \\
\text { range } \\
(\mathrm{GHz})\end{array}$ & $\begin{array}{c}l_{2} \\
(\mu \mathrm{m})\end{array}$ & $\begin{array}{c}l_{2} \\
\text { frequency } \\
\text { range } \\
(\mathrm{GHz})\end{array}$ \\
\hline WM-570 & $330-500$ & 876 & $330-410$ & 646 & $380-500$ \\
\hline WM-470 & $400-600$ & 724 & $400-500$ & 541 & $450-600$ \\
\hline WM-380 & $500-750$ & 568 & $500-620$ & 431 & $570-750$ \\
\hline WM-310 & $600-900$ & 491 & $600-740$ & 362 & $680-900$ \\
\hline WM-250 & $750-1100$ & 388 & $750-930$ & 298 & $840-1100$ \\
\hline WM-200 & $900-1400$ & 350 & $900-1090$ & 232 & $1060-1400$ \\
\hline WM-164 & $1100-1700$ & 285 & $1100-1330$ & 192 & $1290-1700$ \\
\hline WM-130 & $1400-2200$ & 220 & $1400-1700$ & 147 & $1650-2200$ \\
\hline WM-106 & $1700-2600$ & 185 & $1700-2050$ & 126 & $1980-2600$ \\
\hline WM-86 & $2200-3300$ & 130 & $2200-2740$ & 98 & $2490-3300$ \\
\hline
\end{tabular}

\section{LINE CHANGE-OVER}

Table 1 shows that, for each waveguide band, the useable bandwidths for both lines, $l_{1}$ and $l_{2}$, show some overlap - i.e. frequencies where either line can be used to achieve satisfactory calibration. For example, in the WM-380 band, since $l_{1}$ can be used from $500 \mathrm{GHz}$ to $620 \mathrm{GHz}$ and $l_{2}$ can be used from $570 \mathrm{GHz}$ to $750 \mathrm{GHz}$, the overlap region where both lines can be used is from $570 \mathrm{GHz}$ to $620 \mathrm{GHz}$ (a bandwidth of $50 \mathrm{GHz}$ ). When the $3 / 4$-wave TRL calibration technique has been used previously at millimeter-wave frequencies [9-11], a line changeover frequency has been selected somewhere within this overlap region (e.g. at a frequency in the middle of the overlap region) so that $l_{1}$ is used as the TRL Line standard at all frequencies up to the changeover frequency and $l_{2}$ is used as the TRL Line standard at all frequencies at and above the changeover frequency.

However, at submillimeter-wave frequencies, it has been found that the change between using the two different line standards introduces a step-change in the measurement results made using a VNA calibrated using this 3/4-wave TRL technique. Such step changes are due to either differences in the waveguide dimensions, or the flange connections for the two line standards, or both. An example of such a step in measured S-parameters is shown in Fig. 1, which shows an $S_{21}$ measurement of a $270 \mu \mathrm{m}$ length of line as a DUT in the WM-250 waveguide band, i.e. from $750 \mathrm{GHz}$ to $1100 \mathrm{GHz}$. Fig. 1(a) and 1(b) show the real and imaginary components of $S_{21}$, respectively. The frequency range where the $388 \mu \mathrm{m}$ line is used as a standard is shown using a blue trace; the frequency range where the $298 \mu \mathrm{m}$ line is used as a standard is shown using an orange trace. The step in the measured S-parameter that occurs at around $880 \mathrm{GHz}$ is due to the change in the use of the two calibration line standards - i.e. changing from using $l_{1}=388 \mu \mathrm{m}$ to $l_{2}=298 \mu \mathrm{m}$ (shown in Table I). Each line will have slightly different dimensional imperfections - in effect, each line provides a different reference impedance which significantly impacts the calibration quality.

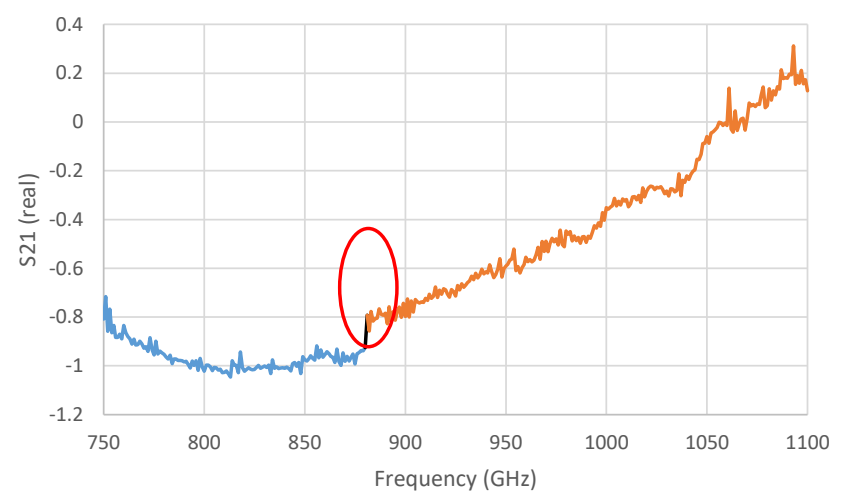

Fig. 1a. Real component of $S_{21}$ for a $270 \mu \mathrm{m}$ line, as a DUT, showing a step change in response at around $880 \mathrm{GHz}$.

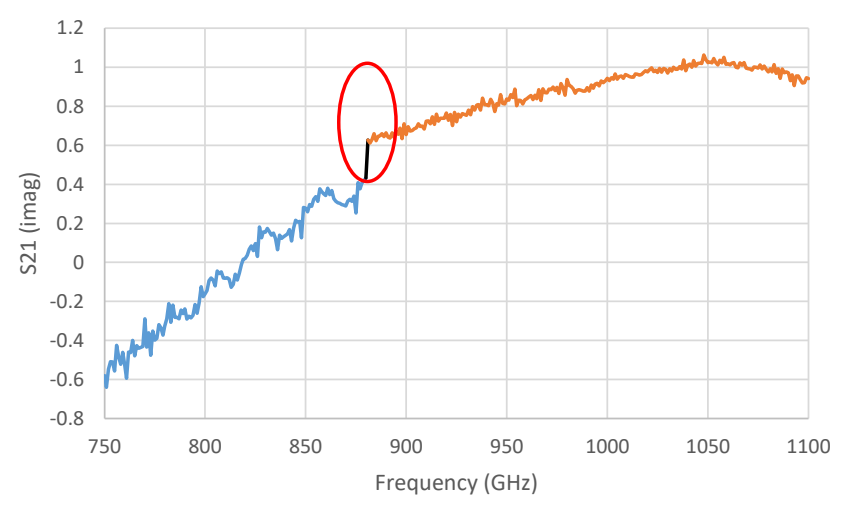

Fig. 1b. Imaginary component of $S_{21}$ for a $270 \mu \mathrm{m}$ line, as a DUT, showing a step change in response at around $880 \mathrm{GHz}$.

To avoid the step change in VNA calibration that occurs at submillimeter-wave frequencies, when changing between different calibration line standards, a combining function is introduced that effectively makes use, to some extent, of data from both lines that are used during calibration. Recognizing that the data from both lines will not be equally wellconditioned at all frequencies (due to the proximity of the phase change due to the line with respect to the calibration failure frequencies) a weighting function is used to combine the two sets of S-parameter results: one set of results derived using $l_{1}$ as the TRL line standard; the other set of results derived using $l_{2}$ as the TRL line standard.

It was recognized in $[14,15]$ that measurement error due to a line's phase difference (with respect to the thru connection) is inversely proportional to the sine of the phase difference. It therefore seems reasonable to base a weighting function around the sine function. In practice, a sine squared weighting function is used here to ensure: (i) the weights are always positive; and (ii) the weight decays rapidly away from the region where the 3/4-wave calibration gives optimum performs - i.e. at the 
frequency corresponding to a $3 / 4$-wavelength. Therefore, at each measurement frequency, the data is weighted according to how well-suited the TRL line standards, $l_{1}$ and $l_{2}$, are for providing data at any given frequency.

First of all, we assign weights, $w_{i}$, such that $0<w_{i}<1$, according to the phase, $\phi_{i}$, of line standard $i(i=1$ or 2$)$ :

$$
w_{i}=\sin ^{2} \phi_{i}
$$

For example: $w_{i}=0$ at $2 n \lambda_{\mathrm{g}} / 4$, and $w_{i}=1$ at $(2 n+1) \lambda_{\mathrm{g}} / 4$ (for $n=0,1,2, \ldots)$, where $\lambda_{\mathrm{g}}$ is the guide wavelength.

We then use a weighted mean, $\hat{x}$, of data, $x_{i}$, from the two lines, at each frequency:

$$
\hat{x}=\frac{\sum_{i=1}^{2} x_{i} w_{i}}{\sum_{i=1}^{2} w_{i}}
$$

where $x_{i}$ is either the real or imaginary component, respectively, of each of the four error-corrected S-parameters.

\section{RESULTS}

Fig. 2 shows the measured $S_{21}$ results for the $270 \mu \mathrm{m}$ length of line, shown in Fig. 1, as a DUT, measured with respect to both TRL calibration line standards (nominal lengths $l_{1}=$ $388 \mu \mathrm{m}$ and $l_{2}=298 \mu \mathrm{m}$ ), before attempting to combine the two sets of measurement results. Fig. 2a shows results for the real component of $S_{21}$ and Fig. 2b shows results for the imaginary component of $S_{21}$.

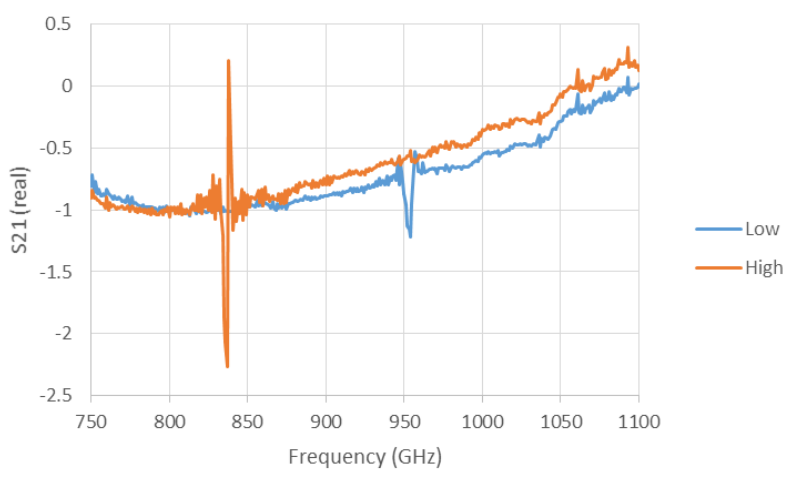

Fig. 2a. Real component of $S_{21}$ for a $270 \mu \mathrm{m}$ line, showing instabilities at approximately $840 \mathrm{GHz}$ and $950 \mathrm{GHz}$.

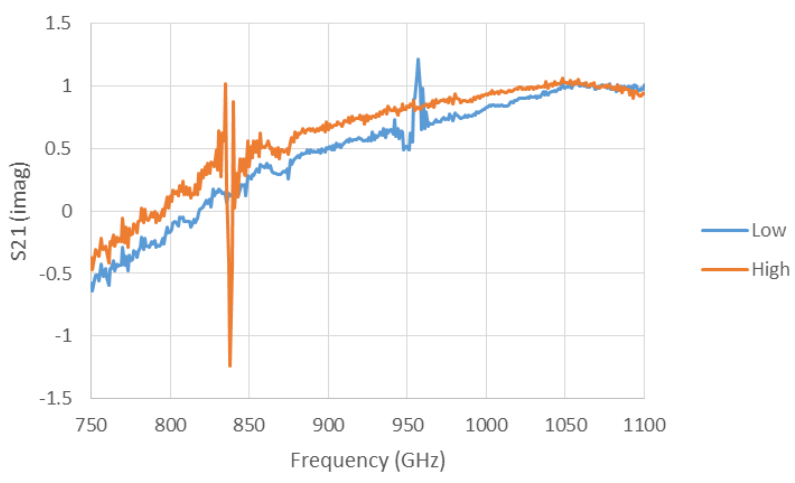

Fig. 2b. Imaginary component of $S_{21}$ for a $270 \mu \mathrm{m}$ line, showing instabilities at approximately $840 \mathrm{GHz}$ and $950 \mathrm{GHz}$.
If the previous method of using just one line as the calibration line standard at each frequency is used, and a changeover frequency in the overlap region is used to combine the two sets of data, the results obtained are as shown in Figs. 1a and 1b (where the line changeover frequency was chosen to be around $880 \mathrm{GHz}$ ). A step is clearly seen at $880 \mathrm{GHz}$ corresponding to the change between the two calibration line standards. This is clearly not attributable to the performance of the DUT and therefore indicates significant measurement error emanating from the calibration process.

Returning to Fig. 2, both traces in each graph show results that are significantly different from each other. There is also clear instability in the results approximately $840 \mathrm{GHz}$, for one set of measurements, and approximately $950 \mathrm{GHz}$, for the other set of measurements. These instabilities correspond to the calibration failure frequencies when the phase change for the line standard passes through either $180^{\circ}$ or $360^{\circ}$.

In practice, it has been found that the frequencies at which these calibrations become unstable do not correspond exactly with the frequencies predicted by phase changes calculated from the measured mechanical length of the lines. This is due to departures in the phase constant of the line, from the classical value, due to effects such as finite conductivity and surface roughness of the internal walls of the waveguide line. Since the S-parameter results of the DUT clearly show the frequencies where the results become unstable, this observation can be used to set the weights to zero at these frequencies.

At the calibration failure frequency for line standard $i(i=1$ or 2), all the $S$-parameters of a device measured with respect to the line show an instability. The calibration failure frequency can be estimated by finding the frequency, $f_{o i}$, at which the linear magnitude of any one of the four $S$-parameters of the device (e.g. $S_{11}$ ) has a maximum corresponding to the instability. To obtain a weighting function for line standard $i$ that vanishes at the observed calibration failure frequency, the weighting function, $w_{i}$, of equation (7) is shifted in frequency as follows

$$
\widetilde{w_{l}}(f)=w_{i}\left(f+\Delta f_{i}\right)=\sin ^{2} \phi_{i}\left(f+\Delta f_{i}\right)
$$

where $f$ is frequency, $\Delta f_{i}=f_{p i}-f_{o i}$ is the difference between the calibration failure frequency predicted from the measured line length, $f_{p i}$, and the observed calibration failure frequency, $f_{o i}$. It is these shifted weighting functions, $\widetilde{w_{l}}$, that are actually used to form the weighted mean in equation (8). In equation (9), the shifted weight function $\widetilde{w_{l}}$ at frequency $f, \widetilde{w_{l}}(f)$, is calculated from the weight $w_{i}$ evaluated at frequency $f+\Delta f_{i}$, $w_{i}\left(f+\Delta f_{i}\right)$, which is in turn calculated from the phase $\phi_{i}$ evaluated at frequency $f+\Delta f_{i}, \phi_{i}\left(f+\Delta f_{i}\right)$.

Fig. 3 shows results obtained after applying the weighting function (i.e. equations (7) and (8)) to the measurement data presented in Fig. 2. It can be seen that the step observed in Fig. 1 is no longer present in the measurement data in Fig. 3. It can also be seen that the results in Fig. 3 vary smoothly, as a function of frequency, across the whole waveguide band and do not contain any of the instabilities seen in Fig. 2 at around $840 \mathrm{GHz}$ and $950 \mathrm{GHz}$. Therefore, Fig. 3 shows that the 
weighting function has been successful in combining the two sets of measurement data for the DUT and producing a physically meaningful set of results for the DUT.

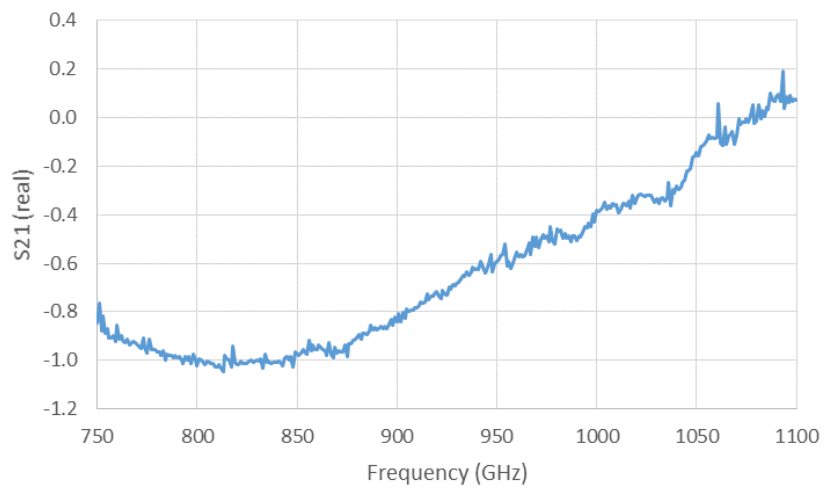

Fig. 3a. Weighted results for the Real component of $S_{21}$ for the $270 \mu \mathrm{m}$ line shown previously in Figs. 1a and 2a

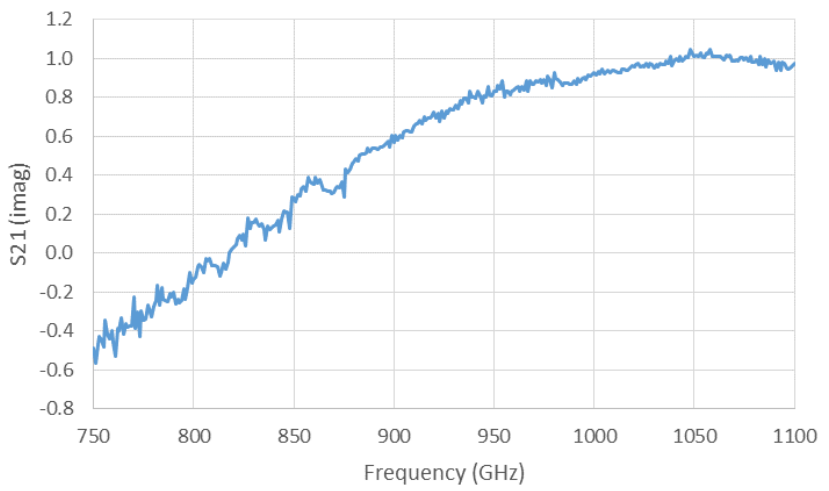

Fig. 3b. Weighted results for the Imaginary component of $S_{21}$ for the $270 \mu \mathrm{m}$ line shown previously in Figs. $1 \mathrm{~b}$ and $2 \mathrm{~b}$

\section{MEASUREMENT UNCERTAINTIES}

When establishing traceable VNA measurements, it is important to estimate the uncertainty in the measurements. At these very high frequencies (i.e. where wavelengths are less than a millimeter) it is expected that random errors due to flange-to-flange alignment during connection will dominate the overall measurement uncertainty. In order to evaluate this effect, consideration is given to the fact that flanges on both a DUT and the VNA test ports will be imperfect, to some extent. For the VNA test ports, this means that it is likely that different measurement results will be obtained for nominally the same device where the differences are due to physical differences (i.e. waveguide aperture size, shape, flange tolerances, etc) between the test ports of port 1 and port 2 of the VNA. Similarly, for a given DUT, it is likely that different measurement results will be obtained when connected to the same VNA test port, if the
DUT is rotated through $180^{\circ}$ prior to connection. (The nature of the waveguide flange for this size of waveguide [16] permits two possible orientations for the connection of a DUT.) If we call one of these orientations 'up' and the other orientation 'down' we can identify four possible connection orientations for a two-port device when connected to a two-port VNA:

1. DUT port 1 connected to VNA port 1 - DUT in 'up' position

2. DUT port 1 connected to VNA port 1 - DUT in 'down' position

3. DUT port 1 connected to VNA port 2 - DUT in 'up' position

4. DUT port 1 connected to VNA port 2 - DUT in 'down' position

Throughout this procedure, port 2 of the device is connected to the other available VNA test port - i.e. VNA port 2, for orientations 1 and 2; VNA port 1, for orientations 3 and 4. By connecting a DUT using the above four orientations, an indication of the contribution to measurement uncertainty due to flange connection variability can be provided by a statistical analysis of the data obtained from each orientation.

For a given two-port DUT, for each $S$-parameter $\left(\mathrm{S}_{i j}, i=1,2\right.$; $j=1,2$ ) at each frequency, we can calculate the mean (where $n$ is the number of connection orientations, $n=4)$ :

$$
\overline{S_{\imath \jmath}}=\frac{1}{n}\left(\sum_{k=1}^{n} \operatorname{Re}\left(S_{i j}\right)_{k}+j \sum_{k=1}^{n} \operatorname{Im}\left(S_{i j}\right)_{k}\right)
$$

and an indication of the uncertainty:

$$
u\left(\overline{S_{l \jmath}}\right)=\sqrt{\frac{\sum_{k=1}^{n}\left|\left(S_{i j}\right)_{k}-\overline{S_{l \jmath}}\right|^{2}}{n(n-1)}}
$$

The modulus signs in the standard uncertainty calculation (equation (11)), cause the standard uncertainty to be a scalar quantity whereas the mean (equation (10)) is a complex-valued quantity. $\quad \overline{S_{l \jmath}}$ is taken as the result of the measurement and $u\left(\overline{S_{l \jmath}}\right)$ is taken as the component of measurement uncertainty due to random errors. A more detailed treatment of the uncertainty in the S-parameters can be obtained by applying the techniques given in $[17,18]$. The process of undertaking these repeated connections also exposes another source of uncertainty - namely, errors due to the flexing of the cables that connect between the VNA front panel and the frequency-multiplier Extender Heads, as shown in Fig. 4. Systematic errors due to the VNA instrumentation (i.e. linearity, noise floor and test port mismatches) can be evaluated and combined in the usual way [19]. Generally, it is found that, at these frequencies, it is the random errors caused by flange misalignment that dominate the overall measurement uncertainty. Table II shows standard uncertainties due to these random errors (i.e. Type-A) along with standard uncertainties due to systematic errors (i.e. Type-B), at selected frequencies for the device whose measurement results are shown in Fig. 3. This table shows that the Type-A uncertainties are much larger than the Type-B 
uncertainties (generally by more than an order of magnitude), confirming that these random errors are the dominant source of measurement uncertainty for waveguide used at these frequencies.

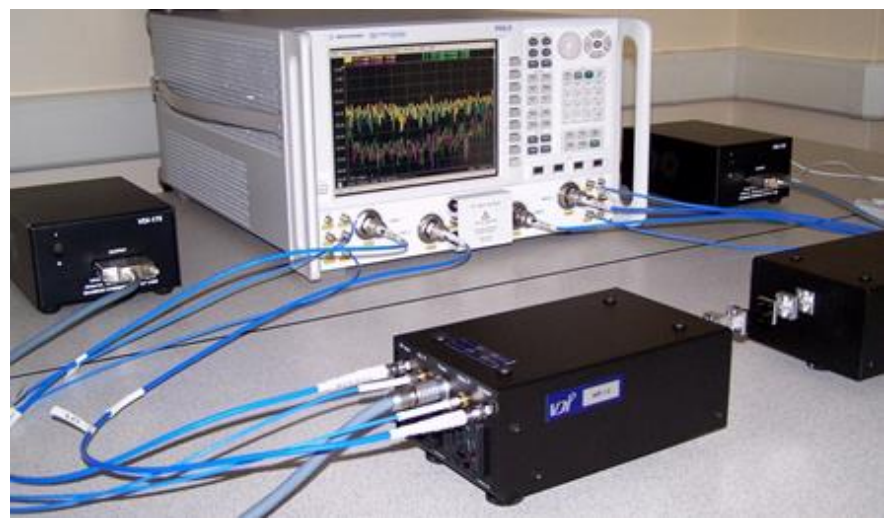

Fig. 4. Submillimeter-wave VNA, showing Extender Heads (foreground) connected to the Display Unit (background) via flexible cables.

TABLE II

TYPE-A AND COMBINED STANDARD UNCERTAINTIES FOR RESULTS SHOWN IN FIG 3 AT SELECTED FREQUENCIES

\begin{tabular}{|c|c|c|}
\hline $\begin{array}{c}\text { Frequency } \\
(\mathrm{GHz})\end{array}$ & $\begin{array}{c}\text { Type-A standard } \\
\text { uncertainty }(\mathrm{dB})\end{array}$ & $\begin{array}{c}\text { Type-B standard } \\
\text { uncertainty }(\mathrm{dB})\end{array}$ \\
\hline 750 & 1.58 & 0.06 \\
\hline 800 & 1.34 & 0.06 \\
\hline 850 & 1.26 & 0.06 \\
\hline 900 & 0.85 & 0.06 \\
\hline 950 & 0.54 & 0.06 \\
\hline 1000 & 0.65 & 0.06 \\
\hline 1050 & 0.91 & 0.06 \\
\hline 1100 & 1.59 & 0.06 \\
\hline
\end{tabular}

\section{CONCLUSION}

This paper has described a strategy for achieving traceable S-parameter measurements using VNAs at submillimeter wavelengths ( 300 to $3000 \mathrm{GHz}$ ). The strategy shows how TRL calibrations can be successfully realized at these very short wavelengths and how the resulting data can be combined using a weighting technique to produce results that show expected physical behavior as a function of frequency. A technique has also been given to help quantify uncertainty due to flange misalignment which is expected to be the dominant source of uncertainty for measurements in waveguide at these frequencies.

\section{REFERENCES}

[1] S. S. Dhillon, et al, "The 2017 terahertz science and technology roadmap", J Phys D: Applied Physics, vol. 50, no. 4, 043001 (49pp), Feb.

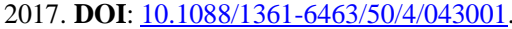

[2] J. D. Albrecht, M. J. Rosker, H. B. Wallace and T.-H. Chang, "THz electronics projects at DARPA: transistors, TMICs, and amplifiers", IEEE
MTT-S Int. Microw. Symp. Dig., May 2010. DOI: $10.1109 /$ MWSYM.2010.5517972.

[3] M. Schroter, et al, "The EU DOTSEVEN project: overview and results", IEEE Compound Semiconductor Integrated Circuit Symposium (CSICS), Oct. 2016. DOI: $10.1109 /$ CSICS.2016.7751070.

[4] Bureau International des Poids et Mesures (BIPM), "The International System of units (SI)", 8th Edition, 2006.

[5] N. M. Ridler, "Traceability to national standards for S-parameter measurements of devices fitted with precision $1.85 \mathrm{~mm}$ coaxial connectors", $68^{\text {th }}$ ARFTG Microw. Meas. Conf., Boulder, Colorado, USA, Dec. 2006. DOI: 10.1109/ARFTG.2006.8361648.

[6] G. J. French and N. M. Ridler, "A primary national standard millimetric waveguide S-parameter measurements", Microwave Engineering Europe, pp. 29-32, Oct. 1999.

[7] G. F. Engen and C. A. Hoer, "Thru-Reflect-Line: an improved technique for calibrating the dual six-port automatic network analyzer", IEEE Trans Microw. Theory Tech., vol. 27, no. 12, pp. 987-993, Dec. 1979. DOI: $10.1109 /$ TMTT.1979.1129778.

[8] C. A. Hoer and G. F. Engen, "On-line accuracy assessment for the dual six-port ANA: extension to nonmating connectors", IEEE Trans Instrum. Meas., vol. 36, no. 2, pp. 524-529, June 1987. DOI: $10.1109 /$ TIM.1987.6312732.

[9] R. G. Clarke, R. D. Pollard, N. M. Ridler, M. J. Salter and A. Wilson, "Traceability to national standards for S-parameter measurements of waveguide devices from $110 \mathrm{GHz}$ to $170 \mathrm{GHz}, 73^{\text {rd }}$ ARFTG Microw. Meas. Conf., pp. 127-136, Boston, MA, USA, June 2009. DOI: $\underline{10.1109 / A R F T G .2009 .5278079}$

[10] N. M. Ridler, R. G. Clarke, M. J. Salter and A. Wilson, "Traceability to national standards for S-parameter measurements in waveguide at frequencies from $140 \mathrm{GHz}$ to $220 \mathrm{GHz}$ ", $76^{\text {th }}$ ARFTG Microw. Meas. Conf., pp. 8-14, Clearwater Beach, FL, USA, Dec. 2010. DOI: $10.1109 /$ ARFTG76.2010.5700046.

[11] N. M. Ridler, R. G. Clarke, M. J. Salter and A. Wilson, "Traceability to national standards for S-parameter measurements in waveguide at frequencies from $220 \mathrm{GHz}$ to $330 \mathrm{GHz}$ ", $78^{\text {th }}$ ARFTG Microw. Meas. Conf., pp. 103-108, Tempe, AZ, USA, Dec. 2011. DOI: $10.1109 /$ ARFTG78.2011.6183882.

[12] N. M. Ridler, "Choosing line lengths for calibrating waveguide vector network analysers at millimetre and sub-millimetre wavelengths", $N P L$ Report TQE 5, National Physical Laboratory, Teddington, UK, Mar. 2009.

[13] IEEE Std 1785.1-2012: "IEEE standard for rectangular metallic waveguides and their interfaces for frequencies of $110 \mathrm{GHz}$ and abovePart 1: frequency bands and waveguide dimensions". DOI: $10.1109 /$ IEEESTD.2013.6471987.

[14] C. A. Hoer, "On-line accuracy assessment for the dual six-port ANA: treatment of systematic errors", IEEE Trans. Instrum. Meas., vol. 36, no. 2, pp. 514-519, June 1987. DOI: 10.1109/TIM.1987.6312730.

[15] R. B. Marks, "A multiline method of network analyzer calibration", IEEE Trans. Microw. Theory Tech., vol. 39, no. 7, pp. 1205-1215, July 1991. DOI: $\underline{10.1109 / 22.85388}$

[16] IEEE Std 1785.2-2016: "IEEE standard for rectangular metallic waveguides and their interfaces for frequencies of $110 \mathrm{GHz}$ and abovePart 2: waveguide interfaces". DOI: 10.1109 /IEEESTD.2016.7564020.

[17] N. M. Ridler and M. J. Salter, "An approach to the treatment of uncertainty in complex S-parameter measurements", Metrologia, vol. 39, no. 3, pp. 295-302, June 2002. DOI: 10.1088/0026-1394/39/3/6.

[18] N. M. Ridler and M. J. Salter, "Evaluating and expressing uncertainty in high-frequency electromagnetic measurements - a selective review", Metrologia, vol. 51, no. 4, pp. S191-198, Aug. 2014. DOI: 10.1088/0026-1394/51/4/S191.

[19] N. M. Ridler and R. G. Clarke, "Establishing traceability to the International System of units for scattering parameter measurements from $750 \mathrm{GHz}$ to $1.1 \mathrm{THz}$ ", IEEE Trans. Terahertz Sci. Technol., vol. 6, no. 1, pp. 2-11, Jan. 2016. DOI: 10.1109/TTHZ.2015.2502068. 


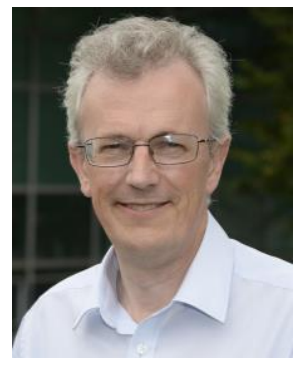

Nick Ridler (M'03-SM'06-F'14) received the BSc degree from King's College, University of London, UK, in 1981. He has more than 35 years' experience working in industrial, government and academic research establishments. He is currently Head of Electrical Science at the U.K.'s National Physical Laboratory. He is also NonExecutive Director of LA Techniques Ltd, and a Visiting Professor at the University of Leeds (Pollard Institute), the University of Liverpool (Department of Electrical Engineering and Electronics) and the University of Surrey (Advanced Technology Institute). He is a Fellow of the Institution of Engineering and Technology (IET) and a Fellow of the Institute of Physics (IOP). His main area of interest is high-frequency precision electromagnetic measurement ( $1 \mathrm{kHz}$ to $1 \mathrm{THz})$.

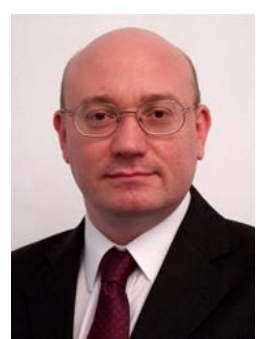

Roland G. Clarke (M'04) was born in Huddersfield, U.K. in 1966. He received the BSc degree from the University of Leeds, Leeds, UK in 2003.

For several years he was responsible for the technical management of highfrequency research laboratories at the University of Leeds. He is currently an Associate Professor within the School of Electronic \& Electrical Engineering at the University of Leeds and a member of the Pollard Institute at the University of Leeds. His research interests are principally concerned with high-frequency metrology, particularly millimeter-wave and submillimeter-wave vector network analyzer measurements.

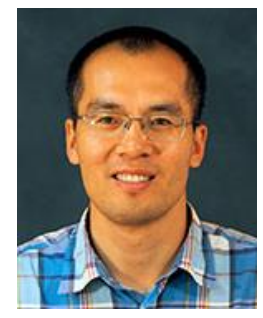

Chong Li (M'12-SM'17) was born in Liaoning, China in 1979. He received the BEng degree from Donghua University, China, in 2002, the MSc degree (Distinction) from the University of Manchester, U.K., in 2007, and the PhD degree in Electronics and Electrical Engineering from the University of Glasgow, U.K., in 2011.

He became a Postdoctoral Research Assistant in 2011 and later a Postdoctoral Research Associate at the University of Glasgow, working on development of millimeter-wave signal sources and terahertz imaging systems. He joined the National Physical Laboratory (NPL), U.K., in January 2014 as a Higher Research Scientist where he contributed to and led several commercial projects and U.K. national and European research projects. He was the measurement service provider (MSP) of the ultrafast waveform metrology service at NPL. He also led work on microwave and millimeter-wave on-wafer measurements. He became a lecturer at the University of Glasgow in August 2017 and is leading the Microwave and Terahertz Electronics Group. His current research interests include microwave and terahertz components, systems and metrology and next generation wireless communications.

Dr. Li held a visiting position at the Advanced Technology Institute (ATI), University of Surrey in 2017. Dr. Li won the best non-student paper prize at LAPC 2015 and has published more than 50 journal and conference papers.

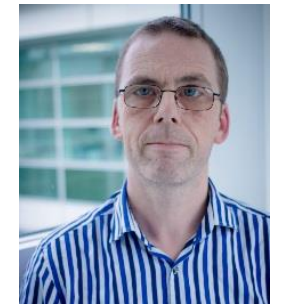

Martin Salter (M '05) received the B.Sc. degree in physics from the University of Manchester and the MSc degree in Microwave Engineering from University College London in 1986 and 1991 respectively. In 1986 he joined the Electromagnetics group at the National Physical Laboratory, Teddington, UK. His main interest is in high frequency electromagnetic measurements. He is a member of the joint University of Surrey/National Physical Laboratory nonlinear microwave measurements \& modelling laboratories (n3m-labs). In 2015 he was a visiting researcher at the Korean Research Institute of Standards and Science, Daejeon, South Korea. 